\title{
Disinfection of bedpans
}

\author{
E. M. DARMADY, K.E. A. HUGHES, J.D.JONES, D. PRINCE, AND PATRICIA VERDON \\ From the Portsmouth and Isle of Wight Area Pathological Service
}

SYNOPSIS A standard dish-washing machine fitted with an automatic cycle has been used to clean and disinfect bedpans. Visual and bacteriological examinations have shown that the machine produces superior and more reliable results than in trials of bedpans submitted to previously described methods.

The bedpan is probably one of the most commonly used articles in ward practice, although little attention has been paid to its cleaning and disinfection. This is partly due to the failure of the medical and administrative staff to penetrate through the portals of the sluice room, and partly to the natural reticence of junior nurses to disclose the sordid nature of their duties. Many of the recommendations made in the past for the cleaning and treatment of bedpans have been devised in the academic circle of committees who are out of touch with the practical aspects and difficulties of nursing procedures. A survey made by the Nuffield Provincial Hospitals Trust (1958) and by Darmady, Hughes, Jones, and Verdon (1959) revealed that many of the pieces of apparatus provided were inadequate or the methods used were so cumbersome that nurses were unable to fulfil the discipline demanded of them.

When this survey began five methods were available.

(1) Washing the bedpan by hand with a mop kept for the purpose and usually stored in one of a series of quite inadequate disinfectants followed by rinsing by hand under running water. As no effort was made to disinfect the pan, it was considered unnecessary to test it for the presence of faecal organisms.

(2) Sluicing the bedpan in a specially constructed piece of apparatus attached to the wall, in which it was sprayed with either cold or hot water and operated by the nurse who lifted one or two levers. No time cycles were recommended and the period of flushing depended on the personal preference of the operator. It was not surprising that a large number of pans showed visible faecal remains and that of the 93 tested all grew faecal organisms.

(3) The bedpan was sluiced by the previous method, but after treatment it was transferred to a boiler usually constructed to hold six or more pans. As the pans were seldom cleaned and as the mass of metal was considerable, the boilers took time to return to boiling water temperature (sometimes over one tour). Furthermore, as the nurse frequently added or removed bedpans during the heating-up of 0 the boiler, it was not surprising that, in the trials carried out, of the 18 treated by this procedure 11 grew faecal organisms.

(4) The bedpan was sluiced as in method 2 , but the apparatus was also fitted with a nozzle for injecting live steam into the chamber holding the pan. As, however, the operation and timing of the various $\stackrel{-}{-}$ procedures were left entirely under the control of a junior nurse the time cycle was reduced to a minimum, and, for subsequent thermocouple studies, the temperature reached by the pan was never adequate to kill faecal organisms. This was confirmed by the fact that out of 48 samples examined faecal organisms were recovered in all cases.

(5) As a number of manufacturers appreciated the inadequacies of personal operation, sluices were devised and fitted with automatic controls and locking doors so that the cycle could not be interrupted. Three different types were tested, their cycles being regulated so that a jet of water either cold or hot and under pressure played over the surface of the pan. The volume of water was sometimes considerable, but in spite of it faecal remains were frequently 윽 observed. This procedure was followed by an injec- $\square$ tion of steam at atmospheric pressure. The cycles varied from 20 seconds to 35 seconds. One of the major disadvantages of this system was that if theces were still adherent to the pan, the steam caused the $\mathbb{O}$ protein to coagulate and baked the residue firmly on to it, making removal well nigh impossible. It was also interesting to note that when such remains were 0 cultivated faecal organisms were isolated. Cultures of the pans showed that the number of occasions on $\stackrel{\oplus}{+}$ which faecal organisms could be isolated was re- 0 duced when compared with the previous methods described, and varied from approximately $25 \%$ to $51 \%$ (Darmady et al., 1959).

These findings were somewhat surprising, and $\frac{2}{0}$ trials therefore were carried out to ascertain what 
temperatures were actually recorded in the pans. In each case the ball of the thermocouple was attached to the rim of the pan by a paper clip. On no occasion in any of the machines using automatic cycles did the temperature reach $80^{\circ} \mathrm{C}$. Further experiments using a machine in which the control of steam could be manually operated showed that at least 35 to 45 seconds were required to reach this temperature, and this can be accounted for by the size of the chamber used to enclose the bedpan and to the mass of metal making up the bedpan itself.

Finally, two further practical disadvantages of this type of machine must be mentioned. First, it is the practice for the nursing staff to carry out a "bedpan round'. The nurse delivers to the various patients a number of pans, and on completion she returns with up to three or four pans for disinfection and subsequent re-use. As they have to be disinfected in these machines singly she is intolerant of the delay caused by the automatic cycle ( $45-50$ seconds), since she may be occupied for four or five minutes awaiting the completion of her load. On occasion this has led to pans used for micturition not being included in the disinfection cycle at all. Secondly, as these machines are covered by specifications laid down by the British Standards Institution the outflow of the soil pipe has an internal diameter of 3 in., and if the nurse has not removed excessive toilet paper and/or sanitary towels, it is frequently blocked. This has necessitated instructions being issued that such objects should be removed by hand before the pans are placed in the machine-a practice which leaves much to be desired. It has been stated that the provision of the restricted outlet was partly due to hospital engineers who felt that it would prevent the blockage of soil pipes at some inaccessible place, and in part that the restriction would lead to a better emptying of the sump. In future an outlet must be provided in such machines which will completely dispose of solids and any sanitary towels.

At about this time, it was noted that the current methods of disinfecting crockery in the tuberculosis wards was hopelessly inadequate (the method employed was similar to that described in 3 above), and that it was possible to culture $M$. tuberculosis from plates artificially infected with tuberculous sputum. In view of the obvious risk to the operator, it was decided to make use of a washing machine fitted with a heater tank. The cycle of the machine was operated by hand, and although this was found to be satisfactory when controlled by a competent person, some operators were unreliable. It was essential, therefore, to make the cycle automatic. The cycle found to be satisfactory was as follows:

(1) The heater tank was set at $190^{\circ} \mathrm{F} .\left(88^{\circ} \mathrm{C}\right.$.).

(2) As soon as the machine was loaded, and the lid closed, approximately one gallon of water at $190^{\circ} \mathrm{F}$. was allowed to enter, and was recirculated by means of a rotating propeller. The mass of crockery cooled the water to below $120^{\circ} \mathrm{F}$. $\left(49^{\circ} \mathrm{C}\right.$.); the optimum temperature for washing varied according to the load used. The chamber was also fitted with a heating coil which heated the recirculated water. As protein coagulates at approximately $140^{\circ} \mathrm{F}$. $\left(60^{\circ} \mathrm{C}\right.$. $)$ it was important that the first, or washing cycle, should not be continued for too long. Investigation with thermocouples showed that the optimum time was between 2 minutes and 2 minutes 30 seconds. In practice the first cycle was set for 2 minutes 15 seconds.

(3) At the end of the washing cycle a valve opened and allowed the water and food debris to run to waste. These were replaced by a further gallon of water at $190^{\circ} \mathrm{F}$. $\left(88^{\circ} \mathrm{C}\right.$.) and allowed to recirculate. As the contents were now clean and at temperatures between $120^{\circ} \mathrm{F}$. and $140^{\circ} \mathrm{F}$. the heat of the water could be raised to ensure disinfection. Experiments showed that this cycle should last six to seven minutes, during the last four of which the temperature of the articles was found to be between $178^{\circ} \mathrm{F}$. and $184^{\circ} \mathrm{F} .\left(81^{\circ}-84^{\circ} \mathrm{C}\right.$.). At the end of the disinfecting cycle the water was allowed to drain away, and since the temperature was high and circulation of air was maintained by means of the rotating propeller the articles were 'flash dried'.

Bacteriological tests using 49 plates showed that when plates were artificially contaminated with Strep. faecalis and put through the machine the organisms were consistently killed.

It occurred to us that such an automatic cycle could be adapted for bedpans. A parallel probably existed in the milk industry which had found that, provided bottles were clean, the relatively low temperature of $180^{\circ} \mathrm{F}$. was adequate to kill pathogenic organisms, including $M$. tuberculosis, but excluding some spore bearers, and to test this hypothesis a basket was constructed to hold four standard bedpans (B.S.I.). Investigations showed that the same automatic time cycle was satisfactory, and since the load was standard in size the temperatures attained were far more reproducible than with crockery.

Accordingly the following procedure was introduced. As soon as the bedpan round had been completed, the solids were discharged into an open sluice or preferably into a sluicing machine fitted with a simple lavatory flush. This takes approximately eight seconds for each bedpan. The bedpan is then transferred to the machine. When the four pans are loaded, the automatic cycle is switched on. Since it is automatic and takes just over nine minutes, the nurse is available for other duties. At the present time 12 such machines have been installed in the Portsmouth Group of Hospitals (three for over two years), and 
two each in the remaining areas of the Hospital Management Committee of the Wessex Region.

Visual investigation showed that all were free from faecal material, and of 184 tested only 11 grew faecal organisms. On each occasion the bacteriological tests were performed as follows:

Sterile 'stick' swabs made with non-absorbent cotton wool were dipped into bijou bottles of sterile nutrient broth. The swabs were then smeared all over the floor and under the rims of the bedpans. On completion the sticks were broken off into the nutrient broth. They were then incubated for seven days and subcultured on to MacConkey agar plates.

Because the object tested was large, and indeed might be contaminated with faecal organisms by an aerosol after heat treatment, particularly as the tests were carried out in the sluice room, these results appear satisfactory. But, it should be pointed out that with this technique it is not possible to determine the total bacterial count as a single colony may still provide a positive result.

\section{DISCUSSION}

The provision of a central sterile supply of bedpans has not so far been considered practical, principally because of the distribution involved, nor does the use of disposable bedpans appear attractive, largely because of cost. Chemical disinfection of bedpans is also unsatisfactory, since it is necessary to emulsify the faeces thoroughly before treatment. Again this is not practical, and with disinfectants such as lysol can be dangerous if the pan is not thoroughly rinsed before re-use. An advantage of the adapted automatic crockery machine is that the apparatus does not require a steam supply, and in the future design of hospitals this may be a great saving in capital cost. At the present time, only about one-third of hospitals in the Wessex Region are equipped with steam to the sluice rooms. Indeed one of the major hospitals in the Group (510 beds) is deficient in this respect. Further, as the machine is an adaptation of a standard manufactured article, the cost is relatively lower than the completely automatic wall type of bedpan sterilizer fitted with steam. Indeed the use of hot water only reduced the risk of faecal remains being baked onto the pan, and the fact that the adapted machine handles four at once has found favour with overworked junior nurses. It is important that the bowl should be filled by water level rather than by time cycle since any variation in water pressure may cause the washing and disinfection treatment to be invalidated.

Some may prefer to add a second washing cycle before the final disinfecting stage. With such machines this should not present mechanical diffi- culties, In most of these investigations no detergents 으 or wetting agents were used, bəcause, first, it was $\vec{F}$ essential to ensure that disinfection was wholly a ? result of the heat treatment, and secondly, it was not $\frac{}{C}$ thought that they would be successful since the $\frac{\bar{\sigma}}{\bar{D}}$. majority depend upon the fat-solvent or liquidizing $\frac{\vec{\Phi}}{\vec{\sigma}}$ action to mobilize the contaminating material (this $\cong$ is particularly true of detergents designed for $\%$ washing crockery). Faecal material, because of its $\vec{\circ}$ high protein and low fat content, is particularly difficult to remove. Another difficulty encountered $\vec{\omega}$ has been the cleaning of pans after faecal material $\stackrel{2}{2}$ has been allowed to stay in them for some hours oro they are contaminated with blood. Trials were made $\vec{A}$ by lining the pan with paper or tissue. However, prolonged contact resulted in the faeces soaking 8 through such materials and becoming adherent to 0 the pan. The only satisfactory method of retaining faeces for visual inspection and yet allowing the $c$ bedpan to be easily sterilized, has been to ask the patient to void urine first and then to line the pan with polythene sheeting. The faecal material can then be removed from the pan by lifting the four $\vec{\varnothing}$ corners of the sheeting, and transferring it to a large $\stackrel{-}{-}$ wax carton, which can subsequently be burnt.

The major disadvantage of the adapted crockery washing machine is that two processes are involved, with the possible danger of cross-infection during the transference of the bedpan from one piece of $\stackrel{\circ}{\mathbb{Q}}$ apparatus to another. In spite of this, in our hands it has consistently produced cleaner and more $\frac{O}{3}$ hygienic pans than any previously encountered. It is clear that in the future the manufacturers must concentrate on a machine which will allow the major solids to be removed and the pan treated with re-옹 circulated hot water, and will treat four bedpans at $\frac{0}{7}$ the same time in a space no greater than that $\underset{3}{3}$. occupied by a crockery washing machine.

The present design of the bedpan makes it 3 difficult to clean. The overhanging internally음 rotated seat makes it difficult for water even under $\supset$ pressure to penetrate to this area, but a pan of . which the seat is externally rotated would be easier to clean, although it is appreciated that such a model might not be so easy to handle and the $N$ contents might spill while it is being carried. N

We are grateful to the Wessex Regional Hospital Board Research Committee for an expenses grant, and을 providing the thermocouples; and to G. R. Scruby Esq., $\mathbb{D}$

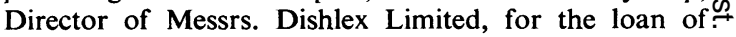
apparatus, and the construction of the special baskets. REFERENCES

Darmady, E. M., Hughes, K. E. A., Jones, I. D., and Verdon, P. E. (1959). Lancet, 1, 622.

Nuffield Provincial Hospitals Trust (1958). Present Sterilizing Practice in Six Hospitals. 